\title{
The human genome: a prospect for paediatrics
}

\author{
R M Gardiner
}

The revolution in molecular biology has generated methods that will almost certainly allow the organisation of the human genome to be understood in detail before the new millenium is more than a decade or two old. A task that seemed unapproachable a few years ago is now seen by optimists as one that might be completed within the foreseeable future. Each month it seems that another disease gene has been mapped, if not cloned, and plans to determine the sequence of all three billion base pairs of the human genome have been made. How has this come about, how will further progress be made, and what are the implications for the health of children?

The scientific methods that have given rise to the 'new genetics', and the implications of this revolution for clinical practice, have been admirably reviewed elsewhere. ${ }^{1}$ It is clear that the scale on which disease is understood has shifted down to the molecular level, and that this has in a sense undermined the old physiological and anatomical boundaries defined by organ systems. This new approach is not surprisingly thrown into sharpest focus in the field of human genetic disease, but its ramifications spread far beyond an increased understanding of some collectively numerous, but individually rare, inherited disorders. Of central importance, nevertheless, is the new capacity to map, and subsequently to isolate, disease genes known only by their phenotype.

This capacity arises from a fusion of classical genetics and molecular biology. In essence, the identification of an unsuspected and vast resource of genetic polymorphism at the DNA level has rendered the human species accessible to linkage analysis, an old genetic tool previously reserved for easily interbred species such as flies and mice.

The mere localisation of genes-gene mapping-is of limited interest. Apart from some clustering of genes into families, there is nothing so far to suggest that the geographical distribution of loci is other than random. In parallel with this new capacity to localise genes, however, a set of methods has arisen that allow genes-once they are localised-to be isolated and characterised. The protein encoded and its defect can then be determined-so called 'reverse genetics'.

The extraordinary revolution that has occurred is best understood by considering briefly our current state of knowledge of the human genome and gene map, the history of linkage analysis, the meaning of genetic polymorphism, and the development of techniques that allow its detection at the level of DNA, achievements in applying these methods to human inherited disease so far, and the prospects for the future.

\section{The human genome}

The human haploid genome comprises about three thousand million base pairs; this is comparatively large. The complete sequence of the simple bacterium Escherichia coli, a mere five million base pairs, has yet to be determined.

As a typical protein comprises say, three hundred amino acids, only one thousand nucleotides are required on average for a structural gene's coding region. The human genome, therefore, has sufficient DNA to encode several million genes. It is probable, however, that no more than $5 \%$ of a mammalian genome codes for protein. The rest is accounted for by highly repetitive sequences with no obvious function, and the introns (non-coding regions between the exons or coding regions), and flanking regions, which make up the main part of individual subject's genes.

It has been estimated that the human genome codes for between 50 and 150000 protein products clustered into 5 to 10000 related families. McKusick's catalogue of mendelian traits has about 4000 entries: gene loci identified by their phenotypes. The chromosomal locations of several hundred of these are known, and the region in which many reside has been identified. About 500 genes have been cloned, but the detailed fine structure of their genomic organisation is known for only a mere handful.

Most of man's genes have not yet been identified-let alone mapped, cloned, and sequenced. The procedure for identifying the gene for a particular protein is relatively straightforward. Antibodies can be used to screen a library of genomic fragments corresponding to regions that are expressed-that is, code for proteins. The corresponding gene can then be identified. Localisation to a particular chromosome and region is then a relatively simple matter. Most disease genes, however, are known only by their phenotype; their biochemical basis is unknown. It is to these disorders that linkage analysis can now be applied. The intention is not merely to map disease loci but, having done so, to isolate the defective gene itself and determine its function and molecular pathology.

\section{Linkage analysis}

Linkage may be defined as the occurrence of two loci sufficiently close to each other on a chromosome that their assortment (that is, distribution to germ cells) is not independent. 
A human cell contains 23 pairs of homologous chromosomes, one of each pair inherited from the father and one from the mother. During meiosis, homologous chromosomes are duplicated and then distributed among four germ cells. Alleles at loci on different chromosomes are inherited together by chance in half of all meioses. Alleles at loci on the same chromosome would always be inherited together if it were not for the phenomenon of recombination, or crossing over.

During meiosis homologous chromosomes repeatedly exchange segments of equal length. This process of recombination, or crossing over, occurs at least once for each chromosome, with perhaps 50 meiotic exchanges in each chromosome set in each generation. Sturtevant, in T H Morgan's laboratory, was the first to recognise that the closer together two loci lie on the same parental chromosome, the less often their alleles are separated by recombination during meiosis (in Morgan's honour his name was given to the unit of genetic distance: loci that recombine in $1 \%$ meioses are said to be one centimorgan (cM) apart). Inheritance patterns of alleles at two loci-linkage analysis-could be used to determine the relative positions of genes along a chromosome, and the first genetic map, of five sex linked loci in drosophila, was produced.

This approach was highly successful in studying mutations in genetic systems that allowed extensive interbreeding of mutant stocks, and complete linkage maps were constructed for several species. Unfortunately its application in man was severely limited for several reasons.

In the human species, information is limited to that arising from random mating through a limited number of generations. This is a severe restraint, but more importantly, mapping of a disease trait requires comparison of its inheritance with that of a genetic marker of known location. Such markers must both be readily detectable, and found in a number of distinguishable variants (polymorphisms) throughout the population. Until recently such markers were limited to a few proteins that displayed limited polymorphism and were representative of only a tiny fraction of the genome. Of course, $\mathrm{X}$ linkage was easily identifiable and a few diseases were mapped by linkage analysis, but for all practical purposes construction of a genetic map was impractical. It was the advent of recombinant DNA technology that transformed linkage analysis into a powerful tool for genetic analysis in man. Genetic polymorphism became detectable at the level of DNA rather than of proteins.

Genetic polymorphism and DNA markers In the terminology of classical genetics a polymorphism is 'the existence in the same habitat at the same time of two or more distinct forms of the species' (E B Ford). In more modern terms it is 'the occurrence of two or more alleles for a particular locus where at least two alleles appear with frequencies of more than one per cent'. At the most basic level, polymorphism reflects a difference in the base sequence at the pair of alleles on homologous chromosomes at a particular locus.

It is now recognised that there are several million single base change differences between the two haploid genome sets found in each cell of an individual subject, a difference in sequence occurring every $200-600$ base pairs. This extensive variation represents an unsuspected vast resource of genetic polymorphism that can easily be detected. Most of these differences occur in 'neutral' DNA, between genes rather than in coding sequences. The sequence changes are said to be 'neutral' because they are passed from generation to generation without any effect on phenotype or on survival.

'Restriction enzymes' provide a practical means of detecting these variations in DNA sequence between homologous chromosomes. A particular restriction enzyme cuts the genome into millions of fragments, not in a random fashion but cutting whenever the base sequence (usually four or six) that defines its restriction site occurs. Variation in the DNA that creates or eliminates a restriction site, or alters the length of DNA between the two particular sites, alters the length of the resulting DNA fragment. This restriction fragment length polymorphism (RFLP) can be detected by Southern blotting.

The problem is the detection of those specific fragments corresponding to the probe in question among the millions generated by digesting genomic DNA. The fragments can be separated according to size by electrophoresis in a gel and 'blotted' on to a sheet of nylon membrane. The filter is exposed to the probe DNA previously labelled with a radioisotope, and this 'hybridises' to fragments bearing the complementary sequence of bases. It is the extraordinary specificity of the hydrogen bonding between complementary base sequences that underlies the power of this method: there are few biochemical reactions with comparable sensitivity and specificity. The location of the hybridised radioactive probe is identified by autoradiography allowing the fragment sizes for an individual subject to be shown.

So that recombination may be detected between a disease locus and a marker locus, the subject in whom the meiosis under study has occurred must be heterozygous at the marker locus. The value of a marker in linkage analysis is therefore critically dependent on how many variants it displays in the population, and therefore the likelihood of a given subject being heterozygous. RFLPs resulting from the presence or absence of a restriction enzyme site exist in only two forms so at least half the population will be homozygous and the meioses studied are often uninformative. More recently a second variety of polymorphism has been identified, which gives rise to multiple allelic variants. In this case the RFLPs arise from variation in the number of 'tandem repeats' of a DNA sequence between restriction sites. For these so called 'variable number tandem repeat' probes the odds that an individual subject will carry different alleles on the homologous chromosomes are much higher. 


\section{Linkage map of the genome using DNA markers}

The first DNA polymorphism that gave rise to a variation in length of restriction fragments was recognised 10 years ago. The potential importance of this observation was soon recognised: the existence of millions of single base change differences between homologous chromosomes could provide a limitless supply of markers scattered throughout the human genome. The approach was set out in detail in $1980 .^{2}$ Several laboratories set out with the deliberate aim of producing a complete linkage map of the human genome using DNA markers. How has this been done? At the outset it was uncertain how many such polymorphic DNA markers it would be possible to identify, and how many would be necessary to cover the genome.

Linkage between markers can be established by following their inheritance in so called 'perfect' families. Maximum information is gained from studying families with four grandparents, two parents, and an average of eight children. An international collaboration was set up in 1984 called the Centre d'Etude de Polymorphisme Humin (CEPH), which maintains cell lines (a renewable source of DNA) on many such families. The Mormon families of Utah have been a particularly fruitful source of such pedigrees.

A huge and expensive effort has been necessary to generate and map the several hundred markers now available. Clones from a 'library' of human genomic DNA are tested to see whether they detect an RFLP. Their inheritance pattern is then studied in the 'perfect' families to identify linkage groups. Linkage groups can be assigned to a specific chromosome if they contain a probe the chromosomal location of which had been previously determined. The actual mapping-determining the arrangement of markers along a chromosome and the distances between them-entails a massive mathematical exercise for which new computer algorithms were designed.

The 'ideal' linkage map would be comprised of highly informative markers spaced evenly at intervals of $10 \mathrm{cM}$ and covering the entire genome. Markers presently available fall somewhat short of this ideal, but few doubt that it will soon be attained. Publication by one research group of details of a collection of markers referred to as 'a genetic linkage map of the human genome' created a furore in 1987. Although it was estimated that this 'map' was detectably linked to $95 \%$ of the DNA in the human genome, the fact remains that several chromosomes contain regions in which the linkage groups are not connected. The intense and public acrimony generated by the publication of this 'map' reflected in part the strong commercial pressures generated in this field. Considerable further work is required to generate a map in which most of the markers are highly informative, and sufficiently close together $(1 \mathrm{cM})$, to provide close enough linkage to act as a starting point for cloning genes.

Disease genes linked to DNA markers Gene mapping in man by linkage analysis has, of course, a long history. Assignment of the genes for colour blindness to the $\mathrm{X}$ chromosome was noted as early as $1911,^{3}$ and a number of diseases genes were subsequently assigned to this chromosome because of their sex linked pattern of inheritance. Progress in the application of DNA methods to localisation of disease genes on the $\mathrm{X}$ chromosome has been rapid, aided of course by the head start of knowing which chromosome to investigate.

The first autosomal linkage in man was reported in $1951,{ }^{4}$ but subsequent progress was slow. The search for DNA markers linked to disease loci that were known to be autosomal has been marked by almost unbelievable luck. For various reasons linkage analysis is easier in dominantly inherited conditions. Linked markers were found among the first few examined in Huntington's chorea ${ }^{5}$ and adult polycystic kidney disease. ${ }^{6}$ Autosomal recessive disorders are a more daunting prospect, as the possibility of non-allelic heterogeneity cannot be discounted in advance. Linked markers have nevertheless been found in at least four such conditions-Friedreich's ataxia, ${ }^{7}$ ataxia telangiectasia, ${ }^{8}$ Batten's disease, ${ }^{9}$ and cystic fibrosis. ${ }^{10}$ The last named is, of course, perhaps the most common and important genetic disorder in white populations. Although, as it happens, the first linkage to cystic fibrosis was found to a protein marker (paraoxonase), localisation was achieved simultaneously by showing linkage to DNA markers on chromosome 7.

Subsequent work has led to mapping of a number of disease genes using this approach including neurofibromatosis, tuberous sclerosis, familial Alzheimer's disease, atopy, and several psychiatric disorders. The problem of non-allelic heterogeneity-identical phenotypes arising from mutations at separate loci-has emerged in some disorders.

Identification of a linked marker does not, of course, hand you the gene. The immediate practical benefit accrues from its use in prenatal, preclinical, and carrier diagnosis. Depending on the recombination frequency between marker and disease gene, the physical distance between the two may be anything between 1 and 10 million base pairs. The task of moving from linked marker to identification of the disease gene itself represents a distinct technical challenge as the method of linkage analysis is no longer applicable at genetic distances so close that recombination is an infrequent event.

\section{Isolation of disease genes}

Genetic linkage analysis allows localisation of a disease gene to within 1 to $10 \mathrm{cM}$, a genetic distance corresponding to a huge physical distance of several million base pairs. Nevertheless, isolation of several disease genes by this approach of 'reverse genetics' has now been achieved. These include Duchenne muscular dystrophy, chronic granulomatous disease, retinoblastoma, von Willebrand's disease, and-most recently and famously-cystic fibrosis. ${ }^{11-13}$

The initial successes were achieved by the ingenious exploitation of cytogenetic alterations identifiable in affected subjects. Clues to the 
precise location of the disease gene were provided by large and identifiable rearrangements of the surrounding DNA. Deletions were particularly valuable.

Pessimists dismissed the isolation of disease genes in which the mutation was a subtle alteration (such as a point mutation) as potentially impossible. They had good reason. An apparently unbridgable 'resolution gap' existed between the scale of linkage mapping information and that encompassed by available cloning methods. Linkage data could identify a region of several million base pairs encompassing the locus of interest, but this could only be sifted through in tiny steps of a few thousand base pairs each-a daunting task for even the most masochistic molecular biologist. Moreover, it was difficult to know in which direction a 'chromosome walk' might be proceeding, and not obvious how one would recognise a particular DNA sequence as a disease gene.

In the last five years several techniques have been developed that bridge this gap. 'Rare cutter' restriction enzymes became available, which cut mammalian DNA infrequently and therefore generate much larger individual fragments. The technique of 'pulsed field' gel electrophoresis was developed, which allowed the size of fragments resolved by electrophoresis to be increased from about 25 kilobases to several million base pairs. A method for cloning the 'ends' of long DNA fragments selectively was devised, which allows probes to be constructed that permit the identification of a genomic fragment at some considerable distance from an initial marker-so called 'chromosome jumping'. Lastly, new vectors (yeast artificial chromosomes-YACs) have been developed that permit cloning of DNA fragments up to a million base pairs in length, substantially larger than was previously possible.

If markers have been identified that 'flank' a disease locus it is therefore now possible to search the intervening region systematically by sequential cloning and analysis until the disease gene is identified. Ultimate success is assured. The researchers know that the gene is there, in the freezer; it is simply a matter of identifying which of the racks of plastic test tubes in there contain it. The power of this approach has been illustrated by the recent isolation of the cystic fibrosis gene. Markers flanking this gene were identified in 1985. Subsequent work narrowed the region of interest to about 1000000 base pairs. The winning teams 'jumped' and walked along 280 kilobases of DNA before the edge of the cystic fibrosis gene was reached, and a further 250 kilobases of DNA had to be isolated and characterised to identify the gene. They were able to show that the mutation causing the disease in $70 \%$ of patients is a 3 base pair deletion that removes a single phenylalanine residue from the encoded protein. This achievement should not be underestimated by comparisons with finding a needle in a haystack. It is analogous to finding a needle dropped anywhere on a 3000 mile highway-the distance from New York to San Francisco.

Isolation of the disease gene then allows the precise molecular genetic pathophysiology of the disease to be determined. Not only can the defective protein product be identified, but the nature of the defect can be analysed in the detail currently available only for the thalassaemias and a few other disorders. This has several important consequences. First, identification of the mutation at the DNA level opens up the possibility of population screening. This will apply only to common disorders, but for diseases such as cystic fibrosis with a gene frequency of one in 20 in white populations the case for screening explains some of the commercial interest in isolation of the gene. Secondly, identification of the protein product and clarification of the biochemistry entailed may allow design of new and rational strategies for treatment.

\section{Future prospects}

The task of mapping, isolating, and characterising all man's genes is immense but finite. It is likely that a high resolution map of linked markers covering the entire human genome will be available within a few years. The suggestion has arisen that, parallel to this effort, the complete base sequence of the human genome-all 3000 million base pairs-should be determined. ${ }^{14}$ The background and implications of this proposal are worth considering.

The nucleotide sequence of a genome represents a physical map at the highest degree of resolution. The scientific justification for wishing to know the entire sequence is uncertain, as it is known that coding genes and their regulatory sequences comprise only about $5 \%$ of the genome. It has been argued therefore that initial effort should go into determining the sequence of the expressed portion, as represented by cDNA clones. It seems likely, however, that the unexpressed regions will contain unsuspected sequences with important functions. Of equal concern is the difficulty in handling the sheer volume of information generated by a complete sequence. So far, about 15 million nucleotides of DNA sequences have been accumulated, of which about 2 million are from human DNA. This latter figure represents only $0.07 \%$ of the total. Storage and analysis of millions of nucleotides of sequence will be a huge task, even if advances in methods occur that allow the rate of analysis to be appreciably increased: with current technology the task would take about 30000 technician years. Cynics have suggested that it would be cheaper to store the sequence in DNA than to extract it from the DNA for storage in computers.

Whether or not the entire sequence is ever determined, it seems likely that physical maps and complete sequences of specific regions or even whole chromosomes will become available within the foreseeable future. What are the implications for paediatrics?

It seems safe to predict that there will be an exponential increase in our understanding of the genetic contribution to disease. The molecular genetic basis of most single gene disorders is now amenable to investigation, and will surely be revealed. In addition to these disease genes, a host (if not all) of the genes that specify a human 
being will be identified with corresponding increases in our understanding of the biology of man. Increased understanding does not of course always lead to practical benefits. A molecular understanding does not guarantee a molecular approach to prevention or cure: sickle cell disease stands witness to that sad fact. Nevertheless it is reasonable to be optimistic that more effective approaches to avoiding these diseases will be developed even if treatment based on our new understanding is more difficult to devise.

Perhaps the most exciting prospect is the possibility of increased understanding of those common disorders that have a genetic component in their aetiology but are not determined by a single gene and may require an appreciable environmental contribution. Complex phenotypes that arise from interactions between several genes, and between genes and the environment, include a number of important childhood conditions such as asthma, epilepsy, diabetes mellitus, and certain congenital malformations. These multifactorial diseases are more difficult to analyse than those attributable to single genes, but the task is no longer impossible. As the number and power of the DNA markers that are available remorselessly increases, these diseases are becoming amenable to investigation. Not only is the number of families required to generate sufficient informative meioses reduced, but entirely new strategies, such as multilocus analysis and simultaneous search, emerge that can overcome-at least in theory-the hitherto insoluble complexities arising in attempts at linkage analysis of multifactorial diseases.

It is indeed ironic that the most profound revolution in biological methods since the invention of the light microscope should coincide with a period of retrenchment in medical research funding. It is no coincidence that a pattern has emerged in which genes are mapped in the United Kingdom, but isolated in the
United States. The latter process is as expensive and labour intensive as formula I motor racing. Winning is difficult if the sponsor will only provide a bicycle.

The 'human genome' today is as much a 'dark continent' as Africa in the early 19th century. Its exploration is about to begin. Genetics has become much too interesting and exciting to leave to the geneticists. Of all the research challenges confronting research minded paediatricians this must surely be the least trivial pursuit.

1 Weatherall DJ. The 'new genetics' and clinical practice. 2nd Ed. Oxford: Oxford University Press, 1985.

2 Botstein D, White R, Skolnick M, Davis RW. Construction of a genetic linkage map in man using restriction fragmen length polymorphisms. Am $\mathcal{F}$ Hum Genet 1980;32:314-31

3 Wilson EB. The sex chromosomes. Archiv fur Mikroskop Anatomie 1911;77:249-71.

4 Mohr J. Estimation of linkage between the Lutheran and the Lewis blood groups. Acta Pathologica et Microbiologica Scandinavica 1951;29:339-44.

5 Gusella JF, Wexler MS, Conneally PM, et al. A polymorphic marker genetically linked to Huntington's chorea. Nature 1983;306:234-9.

6 Reeders ST, Breuning MH, Davies KE, et al. A highly polymorphic DNA marker linked to adult polycystic kidney morphic DNA marker linked to adult polycystic
disease on chromosome 16. Nature 1985;317:542-4.

7 Chamberlain S, Shaw J, Rowland A, et al. Mapping of mutation causing Friedreich's ataxia to human chromosome 9. tion causing Friedreich's

8 Gatti RA, Berkel I, Boder E, et al. Location of an ataxiatelangiectasia gene to chromosome 1lq22-23. Nature 1988; 336:577-80.

9 Eiberg H, Gardiner RM, Mohr J. Batten disease (SpielmeyerSjogren disease) and haptoglobins (HP): indication of linkage and assignment to chromosome 16. Clin Genet 1989; 36:217-8.

10 Wainwright BJ, Scambler PJ, Schmidtke J, et al. Localisation of cystic fibrosis locus to human chromosome 7 cen-q22. Nature 1985;318:384-5.

11 Rommens JM, Ianuzzi MC, Kerem B, et al. Identification of the cystic fibrosis gene: chromosome walking and jumping. Science 1989;245:1059-65.

12 Riordan JR, Rommens JM, Kerem B, et al. Identification of the cystic fibrosis gene: cloning and characterisation of complementary DNA. Science 1989;245:1066-72.

13 Kerem B, Rommens JM, Buchanan JA, et al. Identification of the cystic fibrosis gene: genetic analysis. Science 1989; of the cystic

14 Alberts B. Mapping and sequencing of the human genome. Committee on Mapping and Sequencing the Human Genome. Board on Basic Biology. Commission on Life Sciences, National Research Council. Washington DC: National Academy Press, 1988. 